\title{
Antiplatelet therapy in elderly patients with acute coronary syndrome: Between scientific evidence and future perspectives
}

\author{
Francesco Barillà, Concetta Torromeo, Riccardo lorio, Luigina Porco, Vincenzo Paravati, \\ Carlo Gaudio
}

\author{
Department of Cardiovascular, Respiratory, Nephrologic, Anesthesiologic and Geriatric Sciences, \\ "Sapienza" University of Rome, Italy
}

\begin{abstract}
Dual antiplatelet therapy (DAPT) is an important strategy for reducing cardiovascular events (CV) after an acute coronary syndrome (ACS). Elderly patients undergoing DAPT have a higher risk of bleeding than younger patients for a variety of reasons. Stratification of thrombotic/hemorrhagic risk is mandatory in order to decide on the type and duration of DAPT. The percentage of patients $\geq 75$ years represented in clinical trials is not large, so very often elderly people are prescribed treatment protocols only experimented on younger patients with a lower hemorrhagic risk. However, even in patients aged $\geq 75$ treated with invasive or conservative therapy, after an ACS, a DAPT with aspirin $80-100 \mathrm{mg} /$ day plus a P2Y12 receptor inhibitor for 12 months is recommended. In elderly patients, DAPT should be considered a dynamic process that can be modified over time based on the patient's clinical conditions, or any other necessities (not-procrastinating surgical interventions, comorbidities that can increase hemorrhagic risk). In patients with moderate-high or very high hemorrhagic risk, DAPT treatment should last less than 12 months. A prolongation of DAPT beyond 12 months in this setting is limited to a very low percentage of patients, after careful assessment of ischemic/hemorrhagic profile.
\end{abstract}

Corresponding author: Prof. Francesco Barillà, Department "Cuore e Grossi Vasi A. Reale", Viale Del Policlinico 155, 00161 Rome, Italy.

E-mail: francesco.barilla@uniromal.it

Key words: Acute coronary syndrome; dual antiplatelet therapy; elderly patients.

Received for publication: 24 April 2018

Accepted for publication: 11 May 2018

(C) Copyright F. Barillà et al., 2018

Tipografia PI-ME Editrice, Italy

Monaldi Archives for Chest Disease 2018; 88:952

doi: 10.4081/monaldi.2018.952

This article is distributed under the terms of the Creative Commons Attribution Noncommercial License (by-nc 4.0) which permits any noncommercial use, distribution, and reproduction in any medium, provided the original author(s) and source are credited.

\section{Introduction}

It is estimated that approximately $1 / 3$ of patients with ACS are aged $\geq 70$ years. Advanced age is a good predictor of CV events after an ACS. In patients aged over 75 years the mortality for ACS is about twice than in the younger population. It is estimated that approximately $60 \%$ of overall mortality for ACS is related to elderly patients [1]. Additionally, elderly patients are subject to increases in the number of comorbidities that amplify thrombotic/hemorrhagic risk after ACS. These clinical conditions, in addition to life expectancy and psycho-social factors, affect treatment strategies throughout ACS [2]. Because of this, still many patients do not receive adequate antithrombotic and interventional treatment during ACS, although clinical studies [3] have shown that a more aggressive treatment, whenever possible, has a positive impact on the outcome.

\section{Thrombotic/hemorrhagic risk stratification}

In elderly patient with ACS, the choice and the duration of treatment of P2Y12 receptor inhibitor cannot ignore the assessment of the risk/benefit ratio [4]. In the elderly, some comorbidity increases both ischemic and hemorrhagic risk. Conditions that increase thrombotic risk are: diabetes, $\mathrm{CKD}$, multivessel stenosis or left main disease, peripheral vascular disease etc. Conditions that increase hemorrhagic risk are: CKD, low body weight, history of bleeding, anemia, thrombocytopenia, gastro-duodenopathy, urinary tract disorders, concomitant use of anticoagulant or anti-inflammatory drugs, need for non-postponable interventions, risk of trauma secondary to accidental falls.

To evaluate the thrombotic/hemorrhagic risk, the last Guidelines (GL) recommend the use of two scores, the DAPT and the PRECISEDAPT. The DAPT Risk Score [4] is a numerical value between -2 and +9 , where Higher DAPT scores suggest that the benefit/risk ratio with prolonged DAPT may be favorable. Conversely, lower DAPT scores suggest that the benefit/risk ratio with prolonged DAPT is not favorable. The variables taken into account in this score are: age, the only factor increasing the hemorrhagic risk (65-75 years -1 ; $>75$ years -2$)$; diabetes $(+1)$, smoking $(+1)$, angioplasty or infarction $(+1)$, heart failure or ejection fraction $<30 \%(+2)$ that affect the ischemic risk. The App also calculates the percentage of risk for: myocardial infarction (MI), stent thrombosis (ST), major adverse cardiovascular and cerebrovascular events (MACCE) and bleeding.

The PRECISE-DAPT score takes into account five simple risk factors, which provide a standardized tool for the prediction of out-of-hospital bleeding during DAPT. These variables are: age, creatinine clearance, hemoglobin, white-blood-cell count and previous spontaneous 
bleeding. A longer DAPT significantly increased bleeding in patients at high risk (score $\geq 25$ ), but not in those with lower risk profiles (score $<25)$; $p$ interaction $=0.007$ [4].

Beyond the level of hemorrhagic risk, radial access and III generation DES are strongly recommended from the GL in fragile patients undergoing invasive procedures [4].

\section{Choice of antiplatelet treatment}

Aspirin (ASA), which acts by blocking the formation of thromboxane A2, is certainly the antiplatelet drug to use sine die after an ACS event. ASA reduces the incidence of CV events by $25 \%$ after ACS compared with placebo, with an absolute benefit of 3.6 avoided events per 100 patients at two years of follow-up. The dosage remains $100 \mathrm{mg} /$ day, with a loading dose of $300 \mathrm{mg}$, best to chew and dissolve in the oral cavity. In undertaken, the ASA dose may be $75 \mathrm{mg} /$ day, especially if ticagrelor or prasugrel is used. In frail patients sine die treatment with ASA increases the risk of gastrointestinal events. In patients with ACS and allergic intolerance to ASA treated with invasive strategy, it has been shown that the association of indobufene (reversible COX-1 inhibitor) and clopidogrel is well tolerated and effective in preventing CV events after 18 months of follow-up [5]. Therefore, this drug could be a useful alternative in the elderly in the presence of serious intolerances to the ASA.

The choice on the P2Y12 receptor inhibitor in the elderly patients is more intriguing, between the desire to give a more effective treatment for reducing thrombotic events and the fear to improve the risk of hemorrhagic complications. In this scenario, clopidogrel remains the most recommended drug (class I A) [4].

The evidence of the higher efficacy of prasugrel and ticagrelor compared with clopidogrel in ACS patients has been well demonstrated by TRITON-TIMI 38 and PLATO trials [6-7]. A post-hoc study of TRITONTIMI 38 on elderly patients (about 13\% of population) showed that prasugrel does not have a clear clinical benefit compared to clopidogrel, so prasugrel is not recommended. If specific indications require the use of prasugrel, the loading dosage remains $60 \mathrm{mg}$, with a maintenance dose of $5 \mathrm{mg} /$ day.

In the PLATO study, approximately $15 \%$ of enrolled patients were over 70 years old, some of whom had CKD, others were treated with conservative strategy. So, the use of ticagrelor can be extended to even the most fragile patients. This study showed that also with ticagrelor there is a major exposure to hemorrhagic events (albeit inferior to prasugrel). A prespecified analysis of elderly patients showed that the clinical benefit and safety of ticagrelor was not significantly different between patients $<75$ and those $>75$ years of age [8]. This data was also confirmed by the SWEDEHEART study [9], which enrolled more than 45,000 patients with ACS who were followed for three years, ranging from 59 to 75 years of age (average 67.0), which demonstrated a major efficacy on reducing mortality, re-infarction and stroke, and an increased risk of bleeding events compared to clopidogrel, and this was not different in patients older or younger than 70 years.

Certainly, ticagrelor is the first-choice drug (class II B) in elderly patients where the use of clopidogrel is contraindicate. However, in sight of these data ticagrelor may be the P2Y12 inhibitor recommended even in patients $>70$ years with high risk of MACCE, after careful evaluation of thrombotic/hemorrhagic risk.

Another aspect that encourages the use of ticagrelor in this clinical setting, is that it has been shown that the high on-treatment platelet reactivity (HTPR) increases, for several reasons, with advancing age. In elderly patients the HTPR under clopidogrel is 2-3 times higher than in younger patients and this increases the risk of events in the followup after ACS [10].

The therapeutic management of patients with ACS and CKD is more complex because there is an increase in the risk of cardio-cerebrovascular atherothrombotic events and on the other hand also an increase in the risk of bleeding. In the elderly with $\mathrm{CrCl}>15 \mathrm{ml} / \mathrm{min}$ there are no indications to modify the dosage of antiplatelets. ASA has a renal elimination and although it inhibits renal prostaglandin synthesis, it is still used in severe CKD. Ticagrelor has a biliary elimination, moreover it

Table 1. Clinical and procedural factors that may increase the ischemic/hemorrhagic risk in elderly.

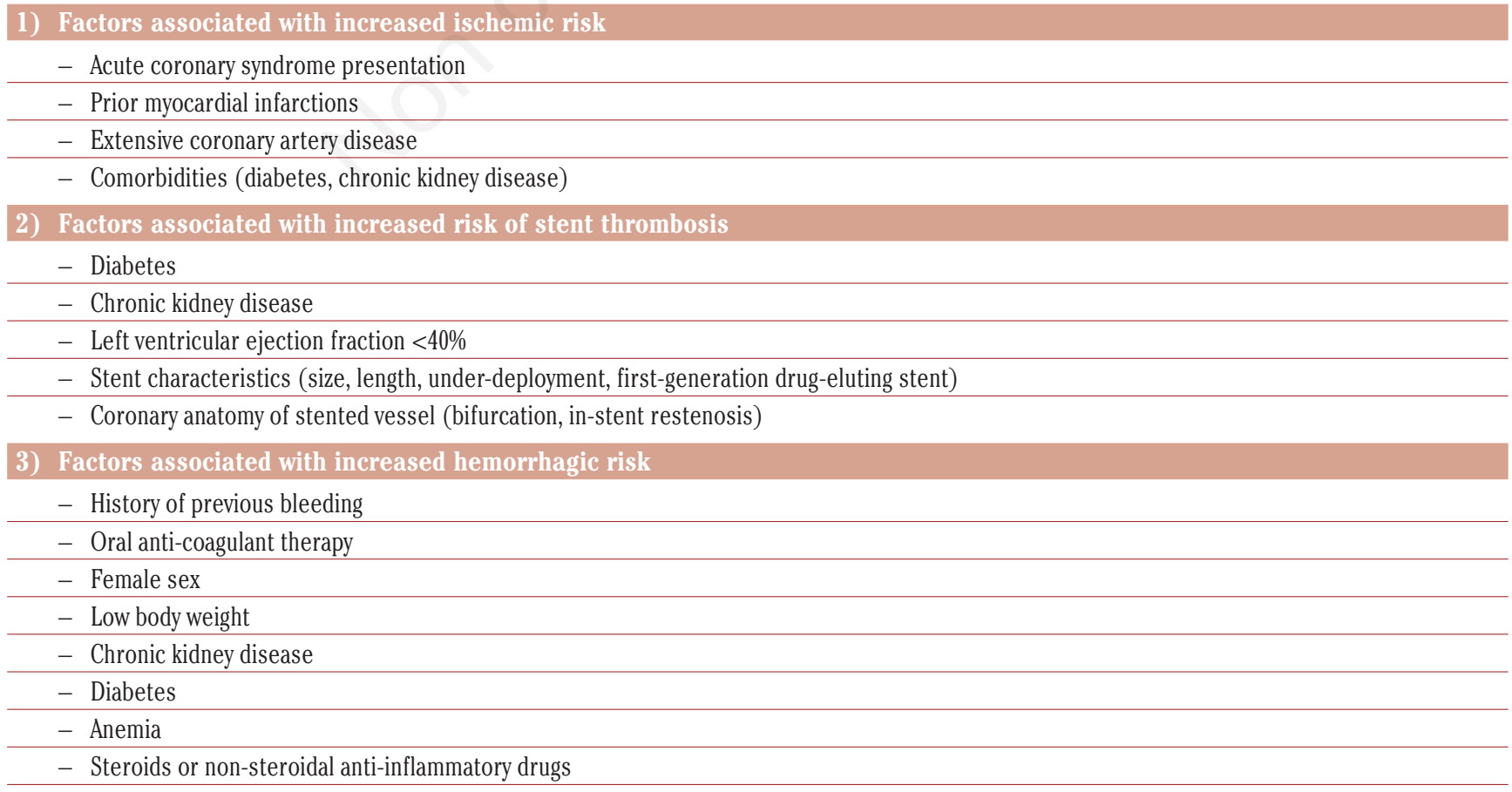


has been shown that this drug is more effective than clopidogrel in patients with CKD. Therefore, DAPT (ASA + ticagrelor) is recommended in patients with $\mathrm{CrCl}>15 \mathrm{ml} / \mathrm{min}$. In the case of severe $\mathrm{CKD}$, with $\mathrm{CrCl}$ $<15 \mathrm{ml} / \mathrm{min}$ (stage V), clopidogrel is recommended.

\section{When to start treatment with DAPT?}

There is no doubt that ASA should be given at the first medical contact. As for the administration of the P2Y12 receptor inhibitor, upstream treatment is recommended in STEMI infarction. GL on STEMI also recommend upstream treatment with DAPT (ASA plus clopidogrel) in elderly patients, both in the case of invasive or pharmacological reperfusion. In the elderly with PCI-treated STEMI, both clopidogrel and ticagrelor are recommended from GL, since there are already wide experiences with these drugs in this scenario (classes IA) [11].

Prasugrel is not recommended in fragile patients: if necessary, the $60 \mathrm{mg}$ dose should be given after coronary angiography and after careful evaluation of hemorrhagic risk.

The upstream use of DAPT does not interfere with the intracoronary use of a glycoprotein IIb / IIla inhibitor. In elderly patients, reversible inhibitors such as tyrofiban or eptifibatide, as bolus injection or, if necessary, infusions of reduced duration (even intraprocedural), are preferred.

There is no unanimous consensus on the upstream use of P2Y12 receptor inhibitors in NSTEMI, especially in the elderly. Such uncertainty also emerges in the GL, which give a recommendation of Class IIa C and report that the loading dose should be administered after the interventional treatment has been programmed [4]. Some evidence suggests that upstream NSTEMI treatment is burdened by an increase in the incidence of bleeding compared to the downstream treatment, especially in elderly patients. In our opinion, upsteam treatment in these patients is indicated if the coronary angiography and therefore a possible PTCA were delayed. In patients that underwent to an early coronary angiography, the up-streamed treatment could be with ASA and heparin, then the clopidogrel or ticagrelor can be administered prior to the PTCA intervention. This strategy would be beneficial if the patient needs a surgical revascularization.

\section{Duration of the antiplatelet treatment}

Even in elderly patient with a NSTEMI or STEMI, there is an indication of a 12 month of DAPT, unless clinical conditions contraindicated [4-11]. The combination ASA-clopidogrel compared to placebo significantly reduced CV death, IM and stroke to 12 months in ACS patients undergoing both invasive and conservative treatments. Since a net clinical benefit was observed also in the elderly subgroup, DAPT is recommended (Class IA) for 12 months after an ACS, regardless of treatment strategy and age of patients. If hemorrhagic risk is increased (PRECISE-DAPT > 25), not rare in the elderly, treatment with DAPT should be reduced to 6 months or even 3 months if the risk is very high (Class IIa B).

Prolonged DAPT over 12 months in elderly patient should be considered after careful stratification of thrombotic/hemorrhagic risk. Clinical evidences in this scenario are still insufficient and certainly this decision must be dynamic with a shortened follow-up [4].

The rationale for a prolonged duration of DAPT beyond 12 months should be the prevention of both stent thrombosis and ischemic events related to other atherosclerotic plaques. Thus, even elderly patients after MI may benefit from a prolonged DAPT beyond 12 months. A position paper from the Italian Federation of Cardiology [12] focused on clinical and procedural factors associated with increased ischemic risk or increased bleeding risk (Table 1). Elderly patients usually have factors for both increased ischemic and bleeding risks, so the decision must be cautious since often the risk of prolonged DAPT overweigh the benefit.

The DAPT study [13] showed that the benefit of DAPT over 12 months in patients with high thrombotic risk was independent of age and type of implanted stent. In fact, significant ST reduction was also observed in patients in whom a III generation stent was implanted.

The PEGASUS-TIMI 54 study [14] enrolled 18.079 patients with previous IM ( $3.083 \geq 75$ years old). The objective of the study was to evaluate the extension of DAPT over 12 months with two doses of ticagrelor: $90 \mathrm{mg}$ and $60 \mathrm{mg}$ bid, compared to placebo. The study showed that both doses in association with ASA were more effective in terms of reduction of composite endpoint (CV death, MI or stroke) compared with placebo. This benefit was more evident in diabetic and in patients with peripheral artery disease. As in the DAPT study, minor and major bleeding (not fatal or hemorrhagic stroke) significantly increases compared to placebo.

\section{Future prospects}

Do we need to overcome ASA or to combine antiplatelet and anticoagulant drugs to further reduce adverse events in elderly patients undergoing antitrombotic treatment after ACS? Currently, all randomized trials have investigated different durations of P2Y12 receptor inhibitors after DES implantation, with ASA continued indefinitely. No clinical studies evaluated the opposite strategy in which ASA was interrupted and the ADP antagonist continued. Accordingly, two international studies (GLOBAL LEADERS and TWILIGHT) are ongoing. On the other hand, the COMPASS study [15] has recently shown that even in elderly patients with stable atherosclerotic disease, rivaroxaban (2.5 mg bid) plus ASA had better CV outcomes than those assigned to ASA alone, although major and minor bleeding increase more than in those assigned to ASA alone. Since this study was performed in stable coronary patients, it is not possible to extrapolate this data in the setting of ACS. However, this study puts the foundation for future research in the field of antithrombotic treatment after ACS.

\section{References}

1. Rosengren A, Wallentin L, Simoons M. Age, clinical presentation, and outcome of acute coronary syndromes in the Euroheart acute coronary syndrome survey. Eur Heart J 2006; 27:789-95.

2. Tarantini G, Berti S, De Luca L, et al. Documento di posizione della Societá Italiana di Cardiologia Interventistica (SICI-GISE): terapia antitrombotica nel paziente anziano con sindrome coronarica acuta. G Ital Cardiol 2016;17(1):64-79.

3. Tegn N, Abdelnoor M, Aaberge L, et al. Invasive versus conservative strategy in patients aged 80 years or older with non-ST-elevation myocardial infarction or unstable angina pectoris (After Eighty study): an open-label randomised controlled trial. Lancet 2016;12;387(10023):1057-1065.

4. Valgimigli M, Bueno H, Byrne RA, et al. 2017 ESC focused update on dual antiplatelet therapy in coronary artery disease developed in collaboration with EACTS. The Task Force for dual antiplatelet therapy in coronary artery disease of the European Society of Cardiology (ESC) and of the European Association for CardioThoracic Surgery (EACTS). Eur Heart J 2017;26:1-48.

5. Barillà F, Pulcinelli FM, Mangieri E, et al. Clopidogrel plus indobufen in acute coronary syndrome patients with hypersensitivity 
to aspirin undergoing percutaneous coronary intervention. Platelets 24;3:183-188.

6. Wiviott SD, Braunwald E, McCabe CH, et al. TRITON-TIMI 38 Investigators. Prasugrel versus clopidogrel in patients with acute coronary syndromes. N Engl J Med 2007;15;357(20):2001-15.

7. Wallentin L, Becker RC, Budaj A, et al. for the PLATO Investigators. Ticagrelor versus Clopidogrel in Patients with Acute Coronary Syndromes. N Engl J Med 2009;361:1045-1057.

8. Husted S, James S, Becker RC, et al. Ticagrelor versus clopidogrel in elderly patients with acute coronary syndromes: a substudy from the prospective randomized PLATelet inhibition and patient Outcomes (PLATO) trial. Circ Cardiovasc Qual Outcomes 2012;5(5):680-8.

9. Sahlén A, Varenhorst C, Lagerqvist B, et al. Outcomes in patients treated with ticagrelor or clopidogrel after acute myocardial infarction: experiences from SWEDEHEART registry. Eur Heart J 2016;21;37(44):3335-3342.

10. Silvain J, Cayla G, Hulot JS, et al. High on-thienopyridine platelet reactivity in elderly coronary patients: the SENIOR-PLATELETstudy. Eur Heart J 2012;33(10):1241-9.
11. Borja Ibanez Stefan James Stefan Agewall, et al. 2017 ESC Guidelines for the management of acute myocardial infarction in patients presenting with ST-segment elevation: The Task Force for the management of acute myocardial infarction in patients presenting with ST-segment elevation of the European Society of Cardiology (ESC). Eur Heart J 2017.

12. Barillà F, Pelliccia F, Borzi M, et al. Optimal duration of dual antiplatelet therapy after percutaneous coronary intervention: 2016 consensus position of the Italian Society of Cardiology. J Cardiovasc Med (Hagerstown) 2017;18(1):1-9.

13. Mauri L, Kereiakes DJ, Yeh RW, et al. DAPT Study Investigators. Twelve or 30 months of dual antiplatelet therapy after drug-eluting stents. N Engl J Med 2014Dec4;371(23):2155-66.

14. Bonaca MP, Bhatt DL, Cohen M, et al. for the PEGASUS-TIMI 54 Steering Committee and Investigators. Long-term use of ticagrelor in patients with prior myocardial infarction. $\mathrm{N}$ Engl $\mathrm{J}$ Med 2015;7;372(19):1791-800.

15. Eikelboom JW, Connolly SJ, Bosch J, et al. COMPASS Investigators Rivaroxaban with or without Aspirin in Stable Cardiovascular Disease. N Engl J Med 2017; 377:1319-1330. 